\title{
Evolution of low mass contact binaries close to the orbital period cut-off
}

\author{
G. Loukaidou and K. Gazeas \\ National and Kapodistrian University of Athens, Department of Physics, \\ Section of Astrophysics, Astronomy and Mechanics, GR 15784 Zografos, \\ Athens, Greece, (E-mail: georgia-louk@hotmail.com)
}

Received: November 1, 2019; Accepted: December 16, 2019

\begin{abstract}
The evolution of eclipsing binaries leads towards angular momentum and mass loss from the systems, due to stellar wind and magnetic braking. Observational investigations of low-temperature and low-mass contact binaries (or LMCBs) in the solar neighborhood provide the means for studying a large sample of such systems. The observed orbital period cut-off limit of 0.22 days is believed to be a result of evolutionary mechanisms, and such systems act as probes in investigating the very evolved stages of systems before their final coalescence. The main goal of this study is the determination of the stellar evolution tracks of these type of LMCBs, which might be correlated to the formation of blue stragglers and rapidly rotating stars.
\end{abstract}

Key words: binaries: contact - stars: physical parameters - stellar evolution

\section{Introduction}

Contact binaries are the most frequently observed type of eclipsing binary system. They are small, cool, low-mass, and occur in old stellar populations. In the framework of the CoBiToM Project a sample of contact binary systems was selected to be studied. The sample was chosen due to their ultra short orbital period $(<0.26 \mathrm{~d})$, some of which exceed the orbital period cut-off of $0.22 \mathrm{~d}$ described by Rucinski (2007). These binary systems, also known as LMCBs (low mass contact binaries), are on the verge of coalescence, as a result of mass and angular momentum loss.

\section{The current study}

The observed light curves have been analysed, and the contact configuration investigated by verifying that both Roche lobes were overfilled. Correlation diagrams were examined (Fig. 1), in order to provide sustainable results and relations between the physical parameters. Thereafter it is possible to obtain solid conclusions, especially in cases when the evolutionary processes are not yet clarified. In the correlation diagrams shown here, the absolute physical parameters 

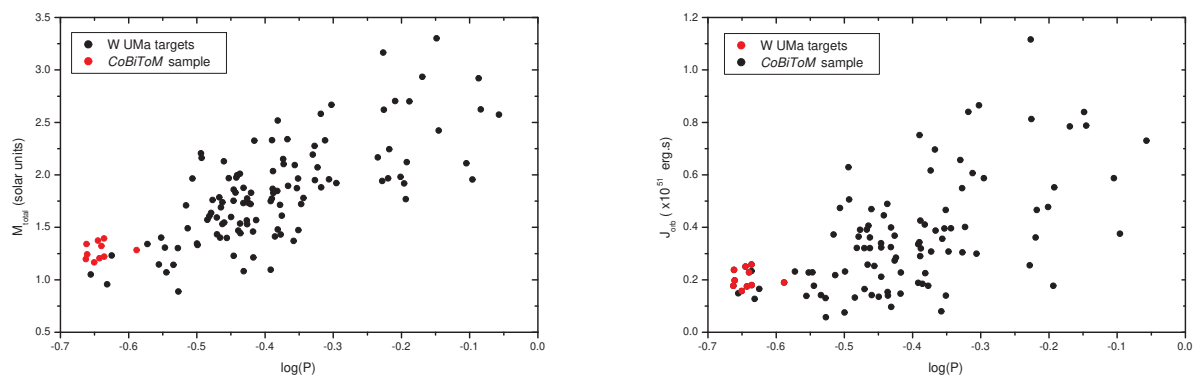

Figure 1. Total mass (left) and angular momentum (right) as a function of orbital period. The sample of CoBiToM Project targets (red circles at lower left of each panel) are shown along the list of W UMa systems with well-defined physical parameters.

of the 10 targets in this study are plotted along with a sample of 112 contact binary systems with well-defined physical parameters from Gazeas et al. (2006). For all 10 systems in our target list we provide high quality four-band photometry, which is essential for stellar models. The number of such systems is very limited and today we know very little about stellar systems close to merging.

Acknowledgements. This research is co-financed by Greece and the European Union (European Social Fund-ESF) through the Operational Programme "Human Resources Development, Education and Lifelong Learning" in the context of the project "Strengthening Human Resources Research Potential via Doctorate Research" (MIS5000432), implemented by the State Scholarships Foundation (IKY). A large volume of the photometric data used in this study were collected in the frame of the photometric observations with the robotic and remotely controlled telescope at the University of Athens Observatory (UOAO) (Gazeas, 2016).

\section{References}

Gazeas, K., The robotic and remotely controlled telescope at the University of Athens Observatory. in , Revista Mexicana de Astronomia y Astrofisica Conference Series, Vol. 48, 22-23

Gazeas, K. D., Niarchos, P. G., Zola, S., Kreiner, J. M., \& Rucinski, S. M., Physical Parameters of Components in Close Binary Systems: VI. 2006, Acta Astron., 56, 127

Rucinski, S. M., The short-period end of the contact binary period distribution based on the All-Sky Automated Survey. 2007, Mon. Not. R. Astron. Soc., 382, 393, DOI: 10.1111/j.1365-2966.2007.12377.x 